\title{
The Norwegian immunisation register - SYSVAK
}

L Trogstad (lill.trogstad@fhi.no) ${ }^{1}$, G Ung $^{1}$, M Hagerup-Jenssen ${ }^{1}$, I Cappelen ${ }^{1}$, I L Haugen ${ }^{1}$, B Feiring ${ }^{1}$

1. Department of Vaccines, Norwegian Institute of Public Health, Oslo, Norway

Trogstad L, Ung G, Hagerup-Jenssen M, Cappelen I, Haugen IL, Feiring B. The Norwegian immunisation register - SYSVAK. Euro Surveill. $2012 ; 17(16)$ :pii=20147. Available online: http://www.eurosurveillance.org/ViewArticle. aspx?Articleld=20147

The Norwegian immunisation register, SYSVAK, is a national electronic immunisation register. It became nationwide in 1995. The major aim was to register all vaccinations in the Childhood Immunisation Programme to ensure that all children are offered adequate vaccination according to schedule in the programme, and to secure high vaccination coverage. Notification to SYSVAK is mandatory, based on personal identification numbers. This allows follow up of individual vaccination schedules and linkage of SYSVAK data to other national health registers for information on outcome diagnoses, such as the surveillance system for communicable diseases. Information from SYSVAK is used to determine vaccine coverage in a timely manner. Coverage can be broken down to regional / local levels and used for active surveillance of vaccination coverage and decisions about interventions. During the 2009 influenza $A\left(\mathrm{H}_{1} \mathrm{~N}_{1}\right)$ pdmog pandemic, an adaptation of SYSVAK enabled daily surveillance of vaccination coverage on national and regional levels. Currently, data from SYSVAK are used, among others, in studies on adverse events related to pandemic vaccination. Future challenges include maximising usage of collected data in surveillance and research, and continued improvement of data quality. Immunisation registers are rich sources for high quality surveillance of vaccination coverage, effectiveness, vaccine failure and adverse events, and gold mines for research.

\section{Background}

Norway has 15 national health registers, including the Norwegian immunisation register, SYSVAK. The national health registers have been established by national health authorities to safeguard nationwide commitments and are legally anchored in $\S 8$ of the Norwegian Law of Health Registers [1]. The registers are nationwide with mandatory notification requirements, and contain exposure and / or outcome data on selected health measures. All registration is based on unique personal identification numbers issued to Norwegian citizens at birth or immigration, which allow linking of different health registers at individual level. The general aim of the Norwegian national health registers is to support health surveillance, research, quality control and improvement of the health system performance.
The Norwegian immunisation register, SYSVAK, is a national electronic immunisation register. Prior to SYSVAK becoming nationwide in 1995 , some counties had already recorded information in its precursor, SYSBARN. SYSBARN was established as a pilot project in 1976 in five of 19 Norwegian counties (Hordaland, Akershus, Hedmark, Oslo and Østfold) [2]. The aims and provisions of SYSVAK are anchored in regulations to the Norwegian health registers act, the SYSVAK regulations [3]. The scope of this paper is to describe the organisation and objectives of SYSVAK and briefly describe the Norwegian immunisation programmes. Data collection, data security measures and confidentiality issues are described, as are routines for access to SYSVAK data for surveillance or research purposes. The use of SYSVAK data for estimating vaccine coverage, surveillance and research are briefly exemplified and future challenges outlined.

\section{Organisation of SYSVAK}

SYSVAK is run and administered by the Department of Vaccines at the Norwegian Institute of Public Health (NIPH) in Oslo. The Department of information technology (IT) and e-medicine is responsible for maintenance and technical adaptations in the register. Healthcare providers in the local municipalities are responsible for administrating the vaccines and for notifying the vaccination to SYSVAK.

\section{SYSVAK objectives}

The original aim of SYSVAK was to register all vaccinations in the Childhood Immunisation Programme for the following purposes:

to maintain an overview of the individual vaccination status of all vaccinees, ensuring that all children are offered adequate vaccination according to the schedule in the Childhood Immunisation Programme and to ensure a high vaccination coverage;

to monitor vaccination coverage for vaccine preventable diseases in the Norwegian population at national, county and municipality levels; and to form a reliable basis for research into the effectiveness and safety of the vaccines in the programme.

Since 2009, SYSVAK has expanded and currently the register includes besides information on all vaccinations in the Childhood Immunisation Programme, all 
human papillomavirus (HPV) vaccinations (including vaccinations given outside the Childhood Immunisation Programme to older girls, boys and adults), and vaccination against all other vaccine preventable diseases (influenza $A\left(\mathrm{H}_{1} \mathrm{~N}_{1}\right)$ pdmo9, seasonal influenza, travel vaccines, etc.). Health professionals are obliged to notify all vaccinations in children and adults to SYSVAK. However, a set of different rules applies regarding the need for consent from the vaccinee or their parent / guardian (Table).

\section{The Norwegian Childhood Immunisation Programme}

The Childhood Immunisation Programme is organised within the public health service. The programme is set at national level and applies to the whole country. The local municipality health services are responsible for delivering the vaccines included in the immunisation programme. The immunisations are provided by public health nurses. General practitioners (GPs) usually play no role in delivering vaccines within the Childhood Immunisation Programme. Infants and toddlers are vaccinated at public health clinics, while school children are vaccinated within the local school health services. Practically all infants and pre-school children in Norway are followed up at the public health clinic, and the majority of Norwegian children are enrolled in public schools, with only $1.7 \%$ attending private schools [4].

Public health clinics and school health services are statutory services that provide health promotion and prevention services for pregnant women, parents, children from birth and adolescents up to 20 years of age. Nurses, doctors and midwives are usually the core staff at a health clinic. The service is a comprehensive offering that includes health checks, vaccinations, health education and counselling free of charge. Given the preventive nature of the service, children with acute or chronic medical conditions are referred to their GP for treatment and follow-up. Special at-risk children, for instance preterm newborns, children of hepatitis B surface antigen (HBsAg)-seropositive (infectious) mothers or those with complicated medical conditions, may receive their first vaccines in the hospital. However, after that, most vaccinations in these children take place at the public health clinic.

All vaccinations within the Childhood Immunisation Programme are free of charge. The uptake of the vaccines in the Childhood Immunisation Programme is monitored by NIPH through the Norwegian immunisation register, and is generally high, between $92-95 \%$ [5]. All vaccinations are offered on a voluntary basis. The vaccines for the programme are distributed free of charge from NIPH to the local vaccine providers, independent of notification to SYSVAK.

\section{The influenza immunisation programme}

In Norway, influenza vaccine is recommended to defined risk groups including elderly aged 65 years or older, through the influenza immunisation programme. The influenza vaccine, as well as other recommended vaccines, is provided both by GPs and through public and private health services [6]. Some vaccinations are also provided by hospitals. Influenza vaccines are not publicly reimbursed.

\section{Data collection}

In general, notifications of vaccinations within the Childhood Immunisation Programme are electronically transferred from the patient record systems to SYSVAK. The vaccinations are entered only once in the electronic patient records (EPR) system, and transferred automatically to SYSVAK. The files are transferred in a standardised $\mathrm{xml-format}$ and the integration module with the EPR systems makes data entry fast and easy for the users.

SYSVAK supports reporting of the following variables:

- personal identification number and name of vaccinee;

- specific code and name of each vaccine;

- batch number of the vaccine;

- date of vaccination for each dose;

- date of notification to SYSVAK for each dose; and

- name and location of vaccinating unit (health clinic, GP, etc).

TABLE

Notification to the Norwegian immunisation register SYSVAK and need for respective consent from vaccinee

\begin{tabular}{|c|c|c|}
\hline Vaccination Programme & Consent & Notification \\
\hline $\begin{array}{l}\text { Childhood Immunisation Programme, } \\
\text { including human papillomavirus }\end{array}$ & Not needed & Mandatory \\
\hline Pandemic influenza & Not needed & Mandatory \\
\hline $\begin{array}{l}\text { Human papillomavirus vaccination of } \\
\text { girls outside the Norwegian Childhood } \\
\text { Immunisation Programme, boys, adults }\end{array}$ & Required & $\begin{array}{c}\text { Vaccinee, parent/guardian can object to } \\
\text { notification }\end{array}$ \\
\hline All other vaccinations & Required & $\begin{array}{l}\text { Oral consent from vaccinee, parent / } \\
\text { guardian sufficient }\end{array}$ \\
\hline
\end{tabular}


All EPR systems are based on the national personal identification number as the unique identifier of the vaccinee. This is also the identifier in SYSVAK. The national personal identification number is verified against the Norwegian population register when the message is transferred to SYSVAK.

Data from public health clinics and school health services are almost entirely ( $>99 \%)$ electronically transferred to SYSVAK (Figure 1). At present, some other public and private health services have also adapted their EPR systems to the integration module. Those who have not installed the integration module supply data to SYSVAK via paper forms or via bulk file transfer. Electronic notification is not yet implemented in most hospitals, and hospital vaccinations of neonates are notified to SYSVAK in collaboration with the child's local health clinic that submits the notification electronically to SYSVAK, or via paper forms. There may be some potential for underreporting but to an extent that is negligible.

\section{FIGURE 1}

Data entry into the Norwegian immunisation register SYSVAK

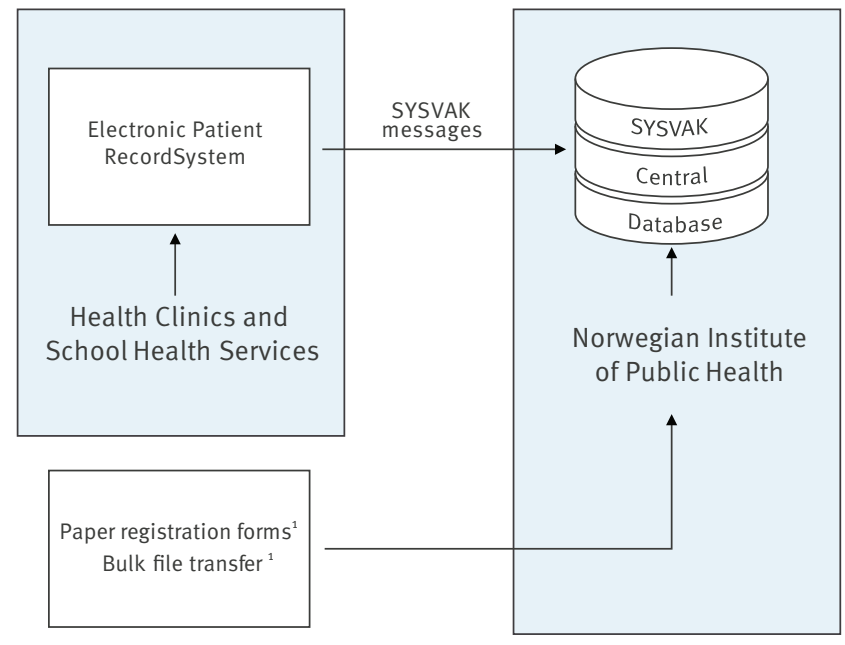

From health clinics, school health services and general practitioners lacking the integration module necessary for electronic transfer of data to SYSVAK.

\section{Access to data and security}

The EPR integration module described above allows healthcare personnel access to search SYSVAK for all vaccines pertaining to one specific vaccinee. This enables active follow-up of the individual immunisation, facilitates timely and correct immunisation, accurate vaccination history, compliance to the programme and completeness of vaccination schedules.

All health information in Norway is regarded as sensitive information, and access to SYSVAK is strictly regulated. Access to SYSVAK is granted on an individual basis via the national health network and all activities are logged. All GPs, hospitals, public health clinics, and other public or private health services are connected via the national health network. This network provides a secure channel for communicating health information in Norway. All health information is encrypted prior to transfer via the network.

Access to data from SYSVAK for research purposes may be granted after application to the NIPH at datatilgang@fhi.no. To ensure adequate data protection and safeguard privacy, permissions from other regulatory bodies like the Regional Committee for Medical Research Ethics or the Norwegian data inspectorate may also be required.

\section{Vaccination coverage}

Vaccination coverage for all vaccines in the Childhood Immunisation Programme is published regularly on the NIPH website [5] and reported annually to the World Health Organization (WHO) and the United Nations International Children's Emergency Fund (UNICEF). The information from SYSVAK can be broken down to regional or municipality levels, allowing active surveillance of vaccination coverage locally and making national or local interventions possible in order to optimise coverage.

Vaccination coverage is defined as the proportion of children within a birth cohort (all children residing in the municipality/county/country as of 31 December) who have been fully vaccinated i.e. who have received all vaccine doses recommended according to the schedule.

As a part of the SYSVAK quality assurance programme, annual reports are sent to the municipality health services. The reports include information on children who are incompletely vaccinated according to age, or have discrepancies in the vaccination schedule. This routine facilitates close follow-up of all children and favours the completeness of the immunisation schedule for each individual, as well as the data quality in SYSVAK.

Vaccination coverage data are based on notification of individual immunisations, and should reflect the true vaccination coverage. When comparing vaccination coverage from Norway with data from other countries using other methods for calculating the coverage, Norway seems to have slightly lower vaccination coverage than for instance Finland and Sweden [7]. This may be due to incomplete notification or delay in the registration, or may reflect that the true vaccination coverage in Norway is lower than in neighbouring countries. However, the rigid criteria in the immunisation register of being vaccinated according to the schedule (i.e. all recommended doses at timely intervals for each specific vaccine) may also be stricter than the criteria used by others in surveys or other methods of estimating and / or reporting vaccination coverage. This may 
again be reflected in slightly lower vaccination coverage estimates in Norway as compared to some other countries.

\section{Examples of vaccination}

coverage in two year-olds

In 2011, 94\% of two year-olds had received three doses of diphtheria-tetanus-acellular pertussis (DTaP) vaccine according to schedule, $95 \%$ had received the Haemophilus influenzae b (Hib) vaccine and $94 \%$ were immunised against poliomyelitis. In total, $93 \%$ of the two year-olds had received the first dose of measlesmumps-rubella (MMR) vaccine. The seven-valent pneumococcal conjugate vaccine ( $\mathrm{PCV}-7$ ) was introduced in Norway in July 2006 and the vaccine was offered to all children born after 1 January 2006. In 2011, 92\% of all two year-olds had received the vaccine according to the schedule (Figure 2).

Improving data quality has been a main focus in SYSVAK during the recent years. Quality assurance of data and close contact with reporting units, public health clinics and school health services have proven crucial for obtaining good quality and completeness of data in the register. A slight increase in overall coverage has been observed during the last ten years and may either reflect this activity, or represent an actual increase in the true vaccination coverage.
The reduction in the uptake of MMR vaccine in two yearolds, especially in 2002 and 2003 (Figure 2), however, reflects a true decrease in coverage. This drop coincided with the debate following the false claims about the link between MMR vaccine and autism put forward by Wakefield et al. in a later retracted paper in the Lancet in 1998 [8]. In Norway, this debate was brought to the public attention in a television programme in the autumn of 2001, and many parents chose not to have their children vaccinated with the MMR vaccine hereafter.

\section{Epidemiological surveillance and research}

The national immunisation register is a valuable tool in epidemiological surveillance, research and quality control. Reliable vaccination data are instantly available and can be accessed for individual ascertainment in outbreak situations, or in cases of suspected vaccine failure or adverse events following immunisation (AEFI). Additional information on outcomes and exposures on individual as well as population levels can be obtained through linkage to other national health registers for vaccine safety and effectiveness studies, as well as studies of attitudes towards vaccination and a number of other research areas.

The use of data from SYSVAK for research purposes has so far been limited, and few scientific papers published $[9,10]$. However, since the influenza $A\left(\mathrm{H}_{1} \mathrm{~N}_{1}\right)$

FIGURE 2

Vaccination coverage in two year-olds who received all recommended vaccines, Norway, 2000-2011

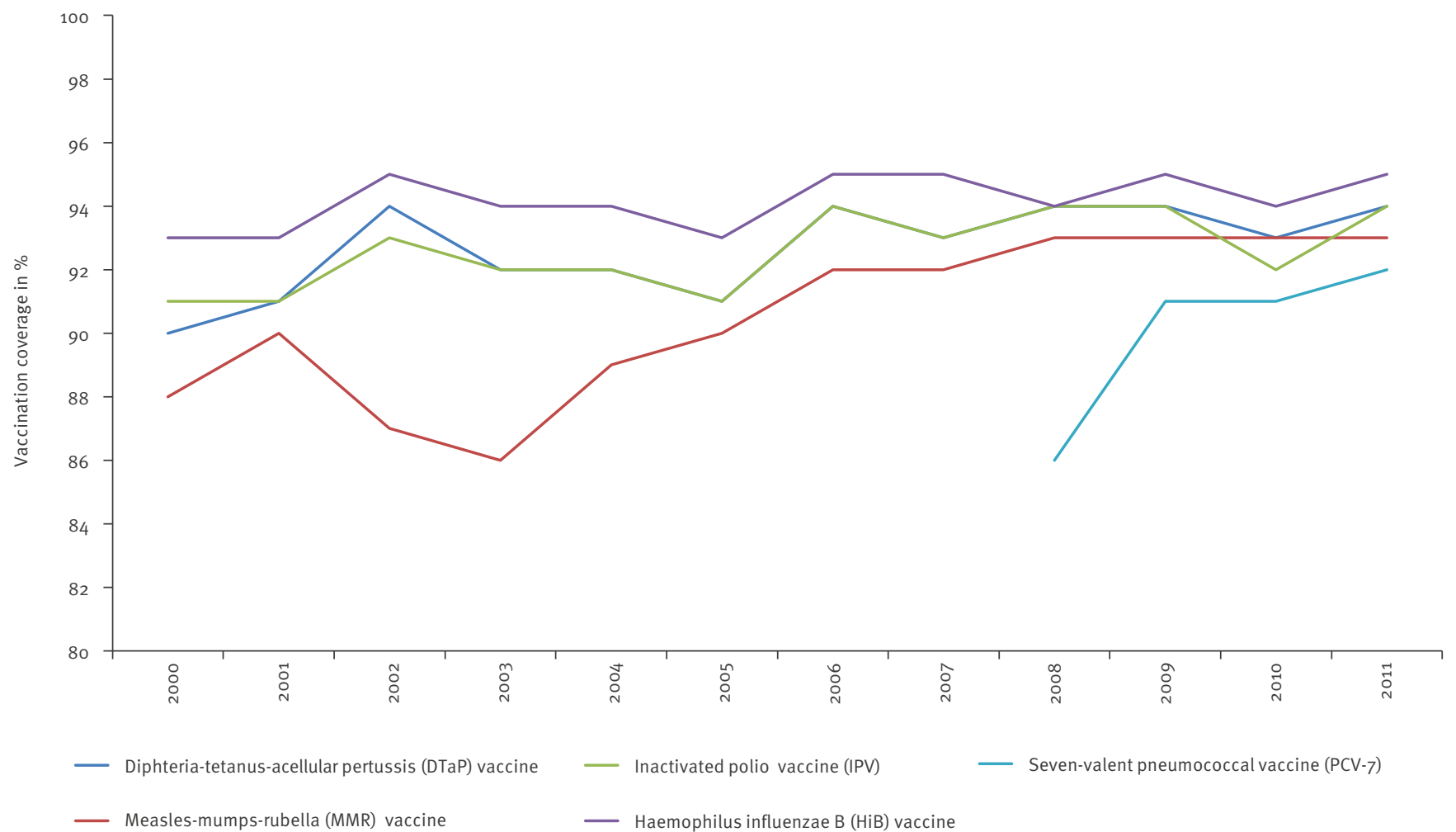

Source: Norwegian immunisation register SYSVAK, Norhealth, 2012. 
pdmog pandemic, there has been a significant increase in applications for access to data from SYSVAK, as described in more detail below. During the years 2006 through 2009 there were five projects using SYSVAK data, whereas in 2010 and 2011 altogether 16 applications for data access from SYSVAK were received.

Measuring the effectiveness of a vaccine programme using SYSVAK data and data linkage

The fact that all registrations into SYSVAK are based on national personal identification numbers allows extensive linkage of information from different sources, both exposure data and outcome data. As an example, PCV-7 was licensed in Norway in 2001, and introduced into the Childhood Immunisation Programme in 2006. The vaccination coverage was monitored using data from SYSVAK, and quickly reached high levels. The effectiveness of the $\mathrm{PCV}-7$ vaccination programme was assessed using (i) data on invasive pneumococcal disease (IPD) incidence obtained from the Norwegian Surveillance System for Communicable Diseases, (ii) pneumococcal serotype distribution obtained from the National Reference Laboratory for Pneumococci, NIPH, Oslo and (iil) vaccination coverage and individual vaccination status from SYSVAK. The incidence rate of IPD among children under two years of age declined rapidly after introduction of the vaccine. The decline was due to a significant fall in incidence rates of vaccine serotype IPD, while no decline in non-vaccine serotype IPD was seen. No cases of vaccine failures were detected $[9,10]$.
SYSVAK and the 2009 influenza A(H1N1)pdm09 pandemic

Notification and access to SYSVAK is usually not permitted via the internet. However, during the influenza $A\left(\mathrm{H}_{1} \mathrm{~N}_{1}\right)$ pdmog pandemic, the NIPH recognised the need to register all vaccinations against pandemic influenza for surveillance purposes and a specific permit was issued to allow easy access to SYSVAK. A specific internet-based application was developed, enabling local healthcare providers to register all vaccinations through a readily accessible web-based interface. Through this system, the notification of pandemic vaccinations was transferred directly to SYSVAK at the time of the vaccination, enabling a day-to-day surveillance of vaccination coverage on national as well as regional levels (Figure 3 ).

According to data from SYSVAK, 1,963,895 individuals received at least one dose of vaccine against 2009 pandemic influenza. The notification to SYSVAK was, however, not entirely complete. Based mainly on the reports on remaining stockpiles in the municipalities, it is estimated that about $90 \%$ of vaccinees were registered in SYSVAK during the pandemic. A provisional estimate by the Norwegian health authorities indicates that 2.2 million people ( $45 \%$ of the population) were vaccinated [unpublished data].

Since the 2009 influenza $A\left(\mathrm{H}_{1} \mathrm{~N}_{1}\right)$ pdmog pandemic, a number of studies have been set up to explore the effects of vaccination or influenza on selected health outcomes, among them pregnancy outcomes and neurological disease. Extensive linkage of register data

\section{FIGURE 3}

Daily administration of vaccine against pandemic influenza A(H1N1)pdm09, Norway, October 2009-January 2010

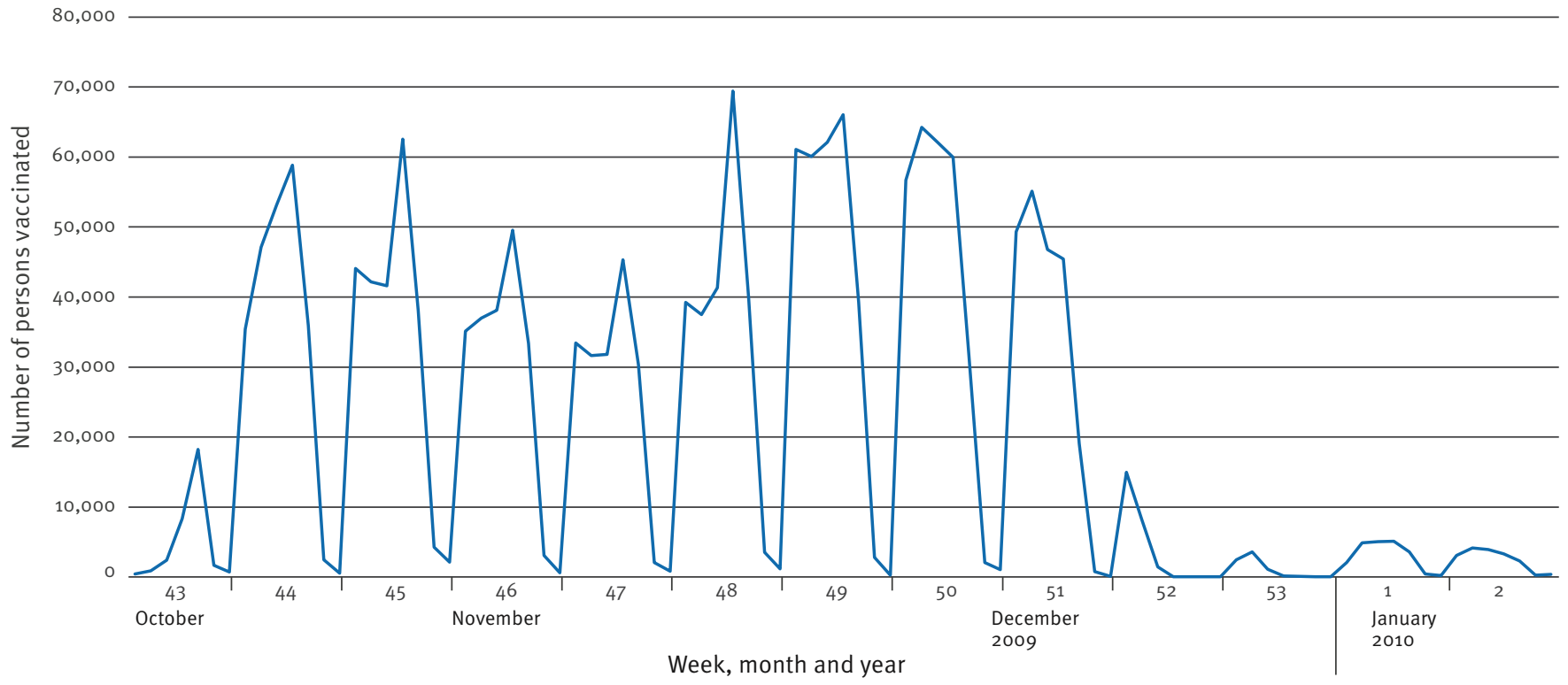


including SYSVAK has been performed for this purpose, and results are still pending.

SYSVAK data are currently also being linked to cohort data from the Norwegian Influenza Cohort, NorFlu. Norflu is a cohort of 3,500 women recruited while pregnant during the 2009 pandemic [11]. The purpose is to study a number of pregnancy outcomes including fetal death, gestational length and birth weight following exposure to pandemic influenza, pandemic vaccination and antiviral medication in pregnancy. Data from SYSVAK is also being linked to the Norwegian Mother and Child Cohort Study, MoBa [12].

Reliable data on vaccination status is crucial in the assessment of AEFIs. Currently, data from SYSVAK are used in studies on adverse events related to pandemic vaccination such as a large national linkage study as well as the European Vaccine Adverse Events Monitoring and Communication (VAESCO) study on narcolepsy following immunisation against pandemic influenza $A\left(\mathrm{H}_{1} \mathrm{~N}_{1}\right)$ pdmo9. A vaccine effectiveness study following immunisation against influenza $A\left(\mathrm{H}_{1} \mathrm{~N}_{1}\right)$ pdmog using SYSVAK data was recently published [13].

\section{Future plans and challenges}

In order to make relevant information available to the public, the Norwegian Ministry of Health and Care Services launched a new web-based initiative in June 2011, allowing the public to access information on their personal health from the national health registers, via www.helsenorge.no and the NIPH website. SYSVAK is one of the first national health registers to enter this service which sets off a new application for the established national health registers. As part of this effort, the service called 'My Vaccines' was recently launched by NIPH.

'My Vaccines' holds records of an individual's vaccinations in Norway, provided that they have been notified to SYSVAK. Access requires a personal log-in procedure after which individuals can download vaccination certificates in Norwegian and English with information on their vaccinations recorded in SYSVAK. Parents can also download vaccination certificates for their children. So far, our experience with the new service is limited.

SYSVAK holds valuable data on vaccinations in Norway. With the aim to maximise the use of collected data in surveillance and research, efforts are needed to use it more actively in monitoring vaccination coverage on individual and group levels. Added value in surveillance of replacement of viral or bacterial strains or in estimating vaccine effectiveness is obtained by linkage of immunisation data to data retrieved from the Surveillance System for Communicable Diseases, which is also run and administered by the NIPH.

Linkage may also be performed to other national health registers, for instance the Cancer Register of Norway, or the Norwegian Patient Register, which holds outcome diagnoses on all in- and out-patient contacts to Norwegian hospitals and specialist healthcare centres. Through such linkages extensive surveillance for a number of vaccine-preventable diseases may be upheld. For instance, we aim to establish a system for systematic surveillance of suspected adverse events following immunisation through linkage between SYSVAK (which holds exposure data on vaccinations) and the Norwegian Patient Register.

A national surveillance programme was set up in Norway following implementation of the HPV vaccine in the Childhood Immunisation Programme [14]. This surveillance programme includes continuous monitoring of vaccination coverage at all levels and effectiveness of vaccination against HPV infection, pre-cancerous cervical lesions and cancer in the female population. Information on vaccination status is retrieved from SYSVAK and will subsequently be linked to outcome data on HPV infection collected in ongoing populationbased prevalence studies, and, at a later stage, to the Cancer Register of Norway for information on pre-cancers and cancers.

We undertake continuous efforts to improve data quality in SYSVAK. Specifically, we aim to complete registration coverage by notification requirements for all vaccinations in children and adults. A further goal is that all notifications should be delivered electronically by EPR integration modules from all public and private health services in Norway for the full set of variables. This would eliminate notification by paper forms and bulk file transfer. To help eliminate paper forms, a webbased interface to SYSVAK for registration via internet is also under development.

To our knowledge, Norway, along with Denmark which will implement a national immunisation register during 2012-13, is one of very few European countries, with mandatory, nationwide immunisation registers based on personal identification numbers [15]. We believe such registers are crucial to provide reliable information on vaccination coverage and adherence to recommended vaccination schedules. Furthermore, immunisation registers are rich sources for high quality surveillance of vaccine effectiveness, vaccine failure and adverse events, and gold mines for future research. We encourage the establishment of immunisation registers in other countries, and continue the efforts to maximise the quality and use of SYSVAK. 


\section{References}

1. Ministry of Health and Care Services. Om lov om helseregistre og behandling av helseopplysninger (helseregisterloven). [Law on health records and processing health information (Health Register Act)]. Oslo: Health and Care Services. [Accessed: 18 Apr 2012]. Norwegian. Available from: http://www.regjeringen. no/nb/dep/hod/dok/regpubl/otprp/19992000/otprpnr-5-1999-2000-/8.html?id=160173

2. Dahl L, Hjørnegaard L. Om 'Sysvak' og 'Sysbarn' -

datasystemer for helsestasjoner. ['SYSVAK' and 'SYSBARN'

- data system for health centers]. Sykepleien 1982;69 (2):14-5. Norwegian.

3. Lovdata. Forskrift om innsamling og behandling av helseopplysninger i Nasjonalt vaksinasjonsregister (SYSVAKregisterforskriften) [Regulations concerning the collection and processing of health data in the national immunization register (SYSVAK Registry Regulations)]. [Accessed 15 Aug 2011]. Norwegian. Available from: http://www.lovdata.no/for/sf/ho/ ho-20030620-0739.html

4. Statistics Norway. Private primary and lower secondary schools. School year 2000/01. Less than 2 per cent in private schools. Oslo: Statistics Norway. [Accessed 29 Mar 2012]. Available from: http://www.ssb.no/privgrs_en

5. Norwegian Institute of Public Health (NIPH). Norwegian Immunisation Registry (SYSVAK). Oslo: NIPH. 29

Mar 2012. Available from: http://www.fhi.no/eway/ default.aspx?pid=238\&trg=MainArea_5811\&MainAr ea_5811=5903:0:15,5305:1:0:0:::0:0

6. Nøkleby H, Feiring B. Det norske vaksinasjonsprogrammet. Tidsskr Nor Lægeforen. 2006;126:2538-40. Norwegian.

7. World Health Organization (WHO). Baccille Calmette Guérin vaccine. Geneva: WHO. 3 Oct 2011. Available from: http:// apps.who.int/immunization_monitoring/en/globalsummary/ timeseries/tscoveragebcg.htm

8. Wakefield AJ, Murch SH, Anthony A, Linnell J, Casson DM, Malik $M$, et al. Ileal-lymphoid-nodular hyperplasia, non-specific colitis, and pervasive developmental disorder in children. Lancet. 1998;351(9103): 637-41.

9. Vestrheim DF, Løvoll $\varnothing$, Aaberge IS, Caugant DA, Høiby $\mathrm{EA}$, Bakke $\mathrm{H}$, et al. Effectiveness of a $2+1$ dose schedule pneumococcal conjugate vaccination programme on invasive pneumococcal disease among children in Norway. Vaccine. 2008;26(26):3277-81.

10. Vestrheim DF, Høiby EA, Bergsaker MR, Rønning K, Aaberge IS, Caugant DA. Indirect effect of conjugate pneumococcal vaccination in a $2+1$ dose schedule. Vaccine. 2010;28(10):2214-21

11. Norwegian Institute of Public Health (NIPH). The Norwegian Influenza Cohort Study (NorFlu). Oslo: NIPH. 18 Oct 2011. Available from: http://www.fhi.no/eway/ default.aspx?pid=238\&trg=MainArea_5811\&MainAr ea_5811=5903:0:15,5331:1:0:0:::0:0

12. Norwegian Institute of Public Health (NIPH). The Norwegian Mother and Child cohort Study. Oslo: NIPH. [Accessed 29 Mar 2012]. Available from: http://www.fhi.no/eway/ default.aspx?pid=238\&trg=MainArea_5811\&MainAr ea_5811=5895:0:15,3046:1:0:0:::0:0

13. Guzman-Herrador B, Aavitsland P, Feiring B, Riise Bergsaker $M A$, Borgen $K$. Usefulness of health registries when estimating vaccine effectiveness during the Influenza $A\left(\mathrm{H}_{1} \mathrm{~N}_{1}\right)$ pdmog pandemic in Norway. BMC Infect Dis. 2012;12(1):63.

14. Norwegian Institute of Public Health (NIPH). HPVnorvaks - National monitoring of HPV vaccination. Oslo: NIPH. 21 Nov 2011. Available from: http://www.fhi.no/eway/ default.aspx?pid=238\&trg=MainArea_5811\&MainAr ea_5811=5903:0:15,5296:1:0:0:::0:0

15. Grove Krause T, Jakobsen S, Haarh M, Mølbak K. The Danish vaccination register . Euro Surveill. 2012;17(17): pii=20155. Available from: http://www.eurosurveillance.org/ViewArticle. aspx?Articleld $=20155$ 